\title{
Comparison of complementary reactions in the production of Mt
}

\author{
S. L. Nelson,,${ }^{1,2}{ }^{*}$ K. E. Gregorich,${ }^{1}$ I. Dragojević, ${ }^{1,2}$ J. Dvořák, ${ }^{1}$ P. A. Ellison, ${ }^{1,2}$ M. A. Garcia, ${ }^{1,2}$ \\ J. M. Gates, ${ }^{1,2}$ L. Stavsetra, ${ }^{1}$ M. N. Ali, ${ }^{2}$ and H. Nitsche ${ }^{1,2}$ \\ ${ }^{1}$ Nuclear Science Division, Lawrence Berkeley National Laboratory, Berkeley, California 94720, USA \\ ${ }^{2}$ Department of Chemistry, University of California, Berkeley, California 94720, USA
}

(Received 20 December 2008; published 25 February 2009)

\begin{abstract}
The new reaction ${ }^{208} \mathrm{~Pb}\left({ }^{59} \mathrm{Co}, n\right){ }^{266} \mathrm{Mt}$ was studied using the Berkeley Gas-filled Separator at the Lawrence Berkeley National Laboratory 88-Inch Cyclotron. A cross section of $7.7_{-3.3}^{+5.2} \mathrm{pb}$ was measured at a compound nucleus excitation energy of $14.9 \mathrm{MeV}$. The measured decay properties of ${ }^{266} \mathrm{Mt}$ and its daughters correspond well with existing data. We compare this experimental result to transactinide compound nucleus formation model predictions, and the previously studied ${ }^{209} \mathrm{Bi}\left({ }^{58} \mathrm{Fe}, n\right){ }^{266} \mathrm{Mt}$ reaction.
\end{abstract}

DOI: 10.1103/PhysRevC.79.027605

PACS number(s): 25.70.Gh, 23.60.+e, 27.90.+b

Reactions with various medium-mass projectiles on nearly spherical, shell-stabilized ${ }^{208} \mathrm{~Pb}$ or ${ }^{209} \mathrm{Bi}$ targets have been used in the investigations of transactinide (TAN) elements and their decay properties for many years. These so-called "cold fusion" reactions produce weakly excited $(10-15 \mathrm{MeV})$ [1] compound nuclei (CNs) at bombarding energies at or near the Coulomb barrier that deexcite by the emission of one to two neutrons.

Until recent years most "cold fusion" type reactions for production of odd- $Z$ TANs used targets of ${ }^{209} \mathrm{Bi}$ instead of ${ }^{208} \mathrm{~Pb}$. The more asymmetric ${ }^{209} \mathrm{Bi}$-based reactions were chosen because they were expected to have a larger cross section for the $1 n$ exit channel product as a result of the lower effective fissility of the reaction [2]. This preference of a slightly more asymmetric system determined which reactions were used in the experimental discoveries of bohrium (Bh, $Z=107$ ) [3,4], meitnerium (Mt, $Z=109$ ) [5,6], roentgenium ( $\mathrm{Rg}, Z=111$ ) [7], and a recent report on the production of $Z=113$ [8].

In addition to the idea that the slightly more asymmetric reactions would give rise to higher cross sections, theoretical predictions have been made about these reactions. Świątecki, Siwek-Wilcyńska, and Wilczyński's "Fusion By Diffusion" (FBD) model [9-11] employs a three step description of heavy element formation by cold fusion reactions. The first step is the sticking, or capture step, where the projectile and target nuclei come into contact and are captured in a mutual nuclear and Coulomb potential minimum. The second step is the "diffusion" along the elongation coordinate, coalescing the target and projectile into a single body. The "survival" of the nucleus by emission of one neutron instead of undergoing fission or other competing deexcitation methods is the final step. Experimentally determined cross sections are typically reproduced within a factor of two by these FBD model predictions [12-15].

In an effort to investigate the role the entrance channel plays in TAN compound nucleus formation, we have undertaken a series of paired reactions which produce the same $\mathrm{CN}$. These paired reactions differ by changing only the location of one proton between the target and projectile nuclei. Recently

*Current address: Lawrence Livermore National Laboratory, P. O. Box 808, L-235, Livermore, California 94551, USA. we have reported on the reaction pairs producing ${ }^{258} \mathrm{Db}$ via the ${ }^{209} \mathrm{Bi}\left({ }^{50} \mathrm{Ti}, n\right)$ and ${ }^{208} \mathrm{~Pb}\left({ }^{51} \mathrm{~V}, n\right)$ reactions [15], and ${ }^{262} \mathrm{Bh}$ via the ${ }^{209} \mathrm{Bi}\left({ }^{54} \mathrm{Cr}, n\right)$ and ${ }^{208} \mathrm{~Pb}\left({ }^{55} \mathrm{Mn}, n\right)$ reactions $[13,16]$. Additionally, the pair of reactions producing ${ }^{272} \mathrm{Rg}$ have been investigated by others. Hofmann et al., at Gesellschaft für Schwerionenforschung (GSI) in Darmstadt, Germany, and Morita et al., at the Institute of Physical and Chemical Research (RIKEN) in Saitama, Japan, have studied the ${ }^{209} \mathrm{Bi}\left({ }^{64} \mathrm{Ni}, n\right){ }^{272} \mathrm{Rg}$ reaction $[7,17,18]$. The complementary ${ }^{208} \mathrm{~Pb}\left({ }^{65} \mathrm{Cu}, n\right){ }^{272} \mathrm{Rg}$ reaction was studied by Folden et al. at the Lawrence Berkeley National Laboratory (LBNL) [19]. With experiments involving paired reactions of elements $Z=105,107$, and 111 now complete, we conclude this study by presenting results on the new ${ }^{208} \mathrm{~Pb}\left({ }^{59} \mathrm{Co}, n\right){ }^{266} \mathrm{Mt}$ reaction studied with the Berkeley Gas-Filled Separator (BGS) at the LBNL 88-Inch Cyclotron. We compare these data with previous results on the ${ }^{209} \mathrm{Bi}\left({ }^{58} \mathrm{Fe}, n\right){ }^{266} \mathrm{Mt}$ reaction $[6,20,21]$.

Meitnerium $(Z=109)$ was discovered as ${ }^{266} \mathrm{Mt}$ by Münzenberg et al. using the cold fusion reaction ${ }^{209} \mathrm{Bi}\left({ }^{58} \mathrm{Fe}, n\right){ }^{266} \mathrm{Mt}$ with the Separator for Heavy Ion reaction Products (SHIP) at GSI [22]. The decay chains observed passed through known nuclides, allowing confident assignment of $Z$ and $A$. The most recent work on ${ }^{266} \mathrm{Mt}$ led by Hofmann et al. in 1997 [21] resulted in a three-point excitation function comprised of 12 decay chains. They fit these three data points with a Gaussian function, obtaining a peak cross section of $7.5 \pm 2.7 \mathrm{pb}$. The observed alpha particle energies of ${ }^{266} \mathrm{Mt}$ vary between $10.48-11.74 \mathrm{MeV}$, which is not unexpected because of its two unpaired particles. GSI reports a half-life of $1.7_{-0.4}^{+0.6} \mathrm{~ms}$ for the decay of ${ }^{266} \mathrm{Mt}$.

The ${ }^{208} \mathrm{~Pb}\left({ }^{59} \mathrm{Co}, n\right){ }^{266} \mathrm{Mt}$ experiment was conducted at the LBNL 88-Inch Cyclotron using the BGS. The BGS separates out evaporation residues (EVRs) from unreacted beam and undesirable reaction products by their differing magnetic rigidities in dilute $\mathrm{He}$ gas, and has been described previously in $[23,24]$. The beam of ${ }^{59} \mathrm{Co}^{13+}$ passed through a $45 \mu \mathrm{g} / \mathrm{cm}^{2}{ }_{-}^{\text {nat }} \mathrm{C}$ carbon window used to separate the vacuum of the beamline from the $67 \mathrm{~Pa}$ of $\mathrm{He}$ fill gas of the BGS and its target chamber. The target wheel consisted of nine arc-shaped targets with a nominal areal density of $\sim 460 \mu \mathrm{g} / \mathrm{cm}^{2}{ }^{208} \mathrm{~Pb}$ metal on 35 $\mu \mathrm{g} / \mathrm{cm}^{2}{ }^{\text {nat }} \mathrm{C}$. A thin $<10 \mu \mathrm{g} / \mathrm{cm}^{2}$ layer of ${ }^{\text {nat }} \mathrm{C}$ was applied to the downstream side of the targets to improve infrared cooling 
and prevent target material loss. The target wheel rotation speed was 5-10 Hz. Calculations of the energy loss through the vacuum window, target, and backing were performed with SRIM-2003 [25,26].

The projectile energy expected to be optimal for production of ${ }^{266} \mathrm{Mt}$ was chosen based on calculations from Świątecki et al.'s "Fusion by Diffusion" (FBD) model $[9,10]$. Experimental masses were used for the target and projectile, and tabulated mass defects from the Thomas-Fermi model [27] were used for those nuclides with unknown masses. The laboratory-frame, center-of-target energy used was $291.5 \mathrm{MeV}$, corresponding to a $\mathrm{CN}$ excitation energy of $14.9 \mathrm{MeV}$. The evaporation residues recoiled out of the target with the momentum of the beam and into the BGS. At the start of the experiment the BGS magnet settings were chosen to guide products with a magnetic rigidity of $2.143 \mathrm{~T} \cdot \mathrm{m}$ to the center of the focal plane detector (FPD). After the first event of ${ }^{266} \mathrm{Mt}$ was detected in strip 45 (near one edge of the FPD), the magnetic field strength was decreased to $2.098 \mathrm{~T} \cdot \mathrm{m}$ in an effort to shift the distribution of products toward the center of the detector. A total separator efficiency of $0.75 \pm 0.02$ was calculated using Monte Carlo simulations of EVR trajectories through the BGS [28].

The detection system used consists of a multiwire proportional counter (MWPC) upstream of the FPD. Signals in the MWPC are primarily used to discriminate implantation-like events such as EVRs from decay-like events such as alpha decays or fissions. The FPD is constructed with a five-sided box geometry from $58 \mathrm{~mm} \times 58 \mathrm{~mm} 300 \mu$ m-thick Si-strip detector cards, each with 16 strips. The focal plane of the BGS is made of three cards totaling 48 strips. These cards are wired at the top and bottom of each strip, so time, energy, and position information may be obtained. The horizontal position is determined by the strip number, and the vertical position is determined by resistive charge division [16]. The sides of the FPD have three cards each on the top and bottom and one card on each side, and are referred to as "upstream" detectors. In addition, another set of three detector cards is placed immediately behind the main focal plane detector cards to detect light ionizing particles such as protons passing through the FPD, and is called the "punchthrough" detector. Additional details about the detector system can be found in previous publications [19]. The alpha particle energy resolution determined by a four-point alpha source over the course of these experiments was $\sigma=26 \mathrm{keV}$. The systematic error in the energy calibration for alpha particles in the FPD was $\pm 5 \mathrm{keV}$, determined by comparing measured and accepted $E_{\alpha}$ from implanted activity after correction for the detector's dead layer and the recoil of the daughter product.

The rate of "EVR-like events" $\left(15.0<E_{\mathrm{EVR}}<30.0 \mathrm{MeV}\right.$ coincident with MWPC signals and anticoincident with punchthrough or upstream signals) during the irradiations had a mean value of $0.26 \mathrm{~s}^{-1}$. The rate of "alpha decay-like events" $\left(7.0<E_{\text {alpha }}<12.0 \mathrm{MeV}\right.$, in the focal plane only, or reconstructed from a focal plane plus an upstream signal, anticoincident with the MWPC and punchthrough signals) had a mean value of $0.04 \mathrm{~s}^{-1}$. ${ }^{266} \mathrm{Mt}$ decay chains were identified by time- and position-correlated decays after an EVR implantation event. A fast beam-shutoff system was implemented to reduce the likelihood of random correlations. Upon the detection of an EVR correlated to an alpha-like event (within $3 \sigma$ of position and $1 \mathrm{~s}$ of the EVR), the beam was automatically switched off for $240 \mathrm{~s}$ to enable registration of any subsequent daughter- or grand-daughter-like decays under strongly reduced background conditions. The data files were analyzed offline, searching for EVR- and alpha-like events within the same energy gates as listed above, and $>80 \mathrm{MeV}$ spontaneous fission (SF)-like events $(80<$ $E_{\text {fission }}<300 \mathrm{MeV}$, no MWPC signal). Once potential decay chains were identified through the offline searches, more specific searches were carried out to lifetimes of $10^{4}$ seconds to try to identify $Z=99-100$ decays with long half-lives.

The known decay properties of ${ }^{266} \mathrm{Mt}$ and its associated daughter products are presented in Fig. 1A. The 291.5 MeV beam used in this study was $0.5 \mathrm{MeV}$ below the threshold for production of the $2 n$ product, ${ }^{265} \mathrm{Mt}$. Accepted decay chains were restricted to an EVR correlated either to a minimum of two full-energy or reconstructed alpha decays, or to an alpha decay followed by an SF. The chain detection efficiency for conclusive identification of ${ }^{266} \mathrm{Mt}$ was calculated to be 0.92 , using the method described in Chap. 2 of [29].

Table I contains a summary of the beam energy, integrated beam dose, and resulting cross section for this work as well as the most recent study by the GSI. Five decay chains attributed to the decay of ${ }^{266} \mathrm{Mt}$ were observed in this work, and these decay chains are depicted in Fig. 1B. Half-life and cross section errors were treated as a special case of the Poisson distribution [30] and our reported error values are at the $68 \%$ confidence interval.

Of the five alpha decays of ${ }^{266} \mathrm{Mt}$ observed in these experiments, only two registered a full-energy signal in the FPD. These decays in chains 1 and 4 registered alpha particle

TABLE I. Summary of observed results in the study of ${ }^{266} \mathrm{Mt}$ by the ${ }^{209} \mathrm{Bi}\left({ }^{58} \mathrm{Fe}, n\right)[21]$ and ${ }^{208} \mathrm{~Pb}\left({ }^{59} \mathrm{Co}, n\right)$ reactions.

\begin{tabular}{lcccccc}
\hline \hline Laboratory & Reaction & $\begin{array}{c}E^{*} \\
(\mathrm{MeV})\end{array}$ & $\begin{array}{c}\text { Target } \\
\text { thickness } \\
\left(\mathrm{mg} / \mathrm{cm}^{2}\right)\end{array}$ & $\begin{array}{c}\text { Dose }\left(10^{18}\right. \\
\text { ions })\end{array}$ & $\begin{array}{c}\text { Number of } \\
\text { events }\end{array}$ & $\begin{array}{c}{ }^{266} \text { Mt cross } \\
\text { section }(\mathrm{pb})\end{array}$ \\
\hline GSI & ${ }^{58} \mathrm{Fe}+{ }^{209} \mathrm{Bi}[21]$ & 13.4 & 0.450 & 1.26 & 5 & $7.4_{-3.3}^{+4.8}$ \\
& 15.4 & 0.450 & 1.27 & 4 & $6.1_{-2.9}^{+4.9}$ \\
& & 16.8 & 0.450 & 2.24 & 3 & $2.5_{-1.4}^{+2.5}$ \\
LBNL & ${ }^{59} \mathrm{Co}+{ }^{208} \mathrm{~Pb}$ & 14.9 & $\sim 0.460$ & $\sim 0.41$ & 5 & $7.7_{-3.3}^{+5.2}$ \\
\hline \hline
\end{tabular}



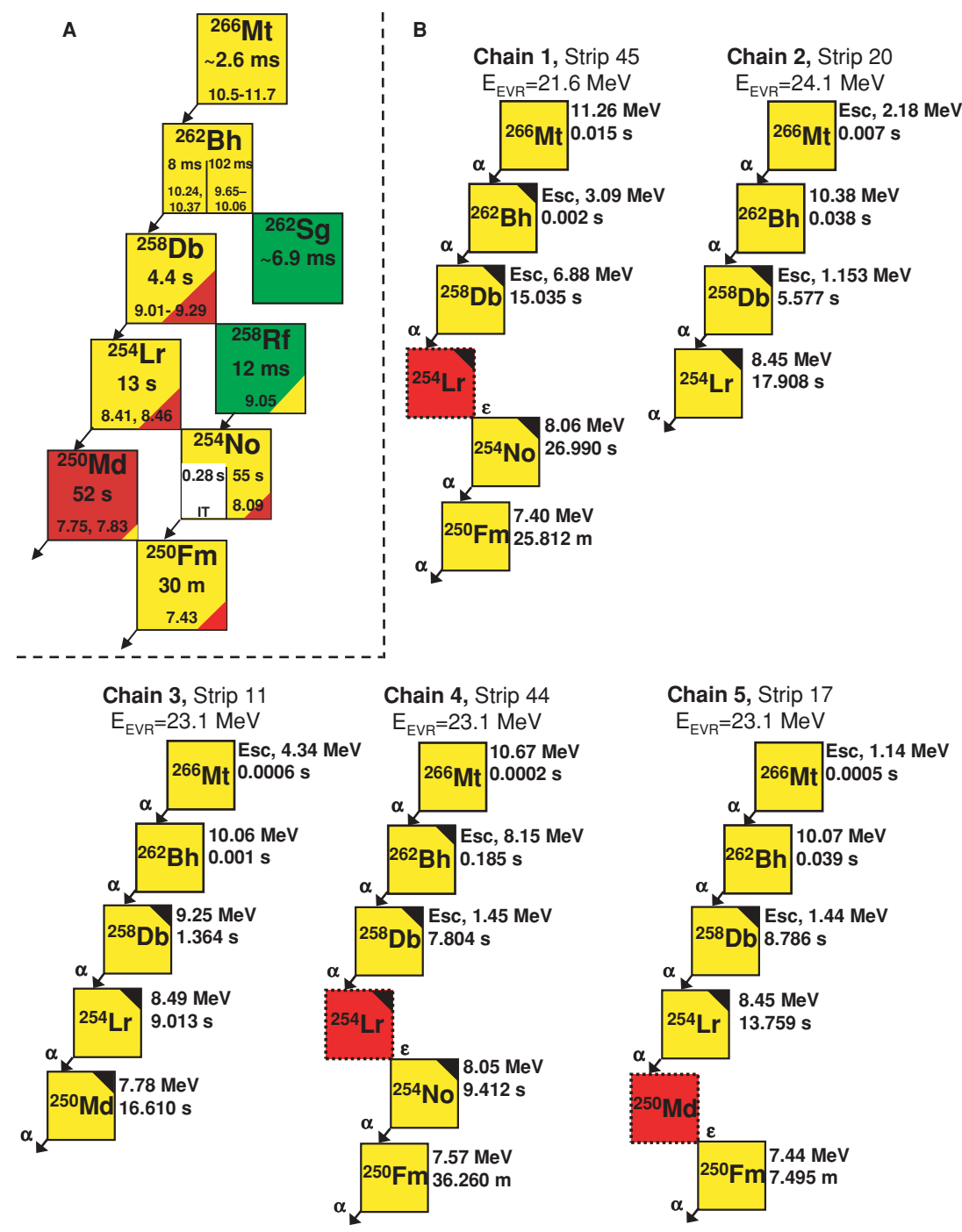

FIG. 1. (Color online) A: Decay properties of ${ }^{266} \mathrm{Mt}$ and its daughter nuclides. Energies are listed in MeV. Data from [4,21,31-39]. B: Decay chains attributed to the decay of ${ }^{266} \mathrm{Mt}$. Black triangles in the upper right corner indicate the beam was turned off. "Esc" denotes alpha particles that exited the focal plane detector and missed the upstream detectors. Lifetimes following EC decay are the sum of the two decays. Dotted border indicates the decay was not directly observed. Colors signify decay the following decay modes: yellow $=$ alpha, red $=\mathrm{EC}$, green $=\mathrm{SF}$. energies of 11.26 and $10.67 \mathrm{MeV}$, respectively, which match the range of alpha energies observed previously [21]. No electron capture (EC) or SF decays attributable to ${ }^{266} \mathrm{Mt}$ were observed in this work, and we report a half-life of $3.3_{-1.0}^{+2.5}$ $\mathrm{ms}$, consistent with the previously reported value of $1.7_{-0.4}^{+0.6}$ $\mathrm{ms}$ [21]. We assign an upper limit of $<0.25$ at the $84 \%$ confidence limit for SF decay, with a corresponding partial SF half-life upper limit of $<0.013 \mathrm{~s}$.

The decay properties of ${ }^{262} \mathrm{Bh}^{\mathrm{g}, \mathrm{m}},{ }^{258} \mathrm{Db},{ }^{254} \mathrm{Lr},{ }^{254} \mathrm{No}$, ${ }^{250} \mathrm{Md}$, and ${ }^{250} \mathrm{Fm}$ agree well with the findings in the literature [31-37]. Figure 1B contains more specific information about individual nuclides.

A calculation of the expected number of randomly correlated decays was performed, using a method similar to the one described in [16]. Because the lifetimes of the ${ }^{266} \mathrm{Mt}$ and ${ }^{262} \mathrm{Bh}$ are short compared to the lifetimes of the decays including ${ }^{258} \mathrm{Db}$ and beyond, calculations were performed that included an EVR correlated to a minimum of one alpha decay within $1 \mathrm{sec}$, and correlated to a minimum of additional one alpha decay within $240 \mathrm{sec}$. Alpha-like events were required to have energies between 7.0-12.0 MeV to cover the range of energies spanned by all products. EVR-like events were required to have energies between 15.0-30.0 MeV. The duration of the experiment was 720,887 seconds. The number of EVR-like events were multiplied by the Poisson probability of observing at least one alpha within $1 \mathrm{sec}$ and the Poisson probability of observing at least one alpha within the subsequent $240 \mathrm{sec}$. The number of random chains expected over the duration of the experiment from one short alpha-like event and one longer-lifetime alpha-like event was 0.095 , and on the order of $10^{-5}$ and lower for EVRs followed by greater than two alpha-like events. Thus, it is statistically likely that the five alpha decay chains observed in this work are true events and not random correlations from unrelated signals.

A cross section of $7.7_{-3.3}^{+5.2} \mathrm{pb}$ was measured at an excitation energy of $14.9 \mathrm{MeV}$ in the ${ }^{59} \mathrm{Co}+{ }^{208} \mathrm{~Pb}$ reaction. Figure 2 illustrates the cross section data from the ${ }^{58} \mathrm{Fe}+{ }^{209} \mathrm{Bi}$ excitation function reported by Hofmann et al. [21] and the ${ }^{59} \mathrm{Co}+{ }^{208} \mathrm{~Pb}$ reaction in this work. The two peak cross section values are the same within statistical uncertainty. Overall, the decay properties of ${ }^{266} \mathrm{Mt}$ and its daughters fit well with the previously reported values by GSI.

Świątecki has predicted $1 n$ cross sections using a reparametrized FBD model [9-11]. This reparametrization 


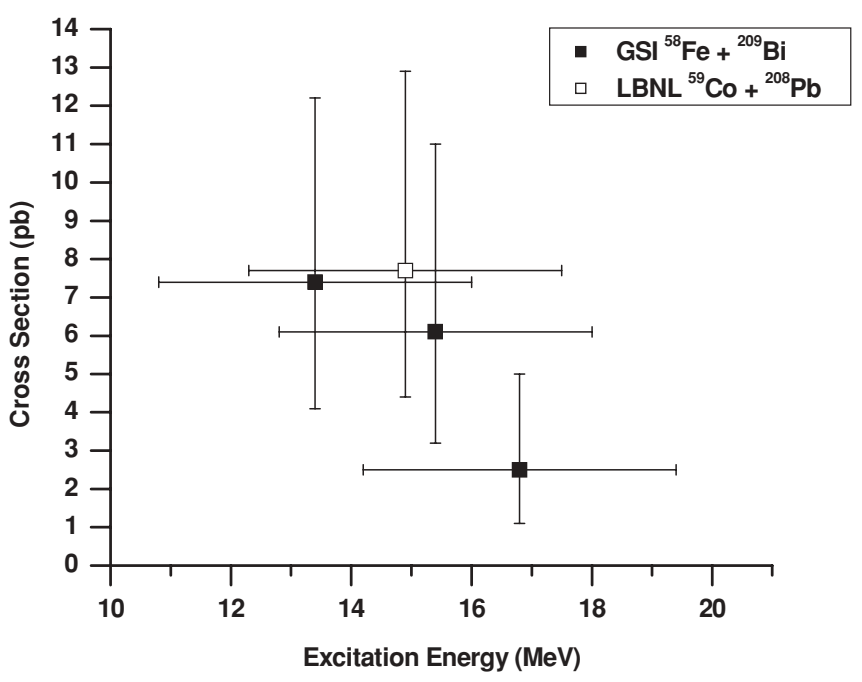

FIG. 2. Experimental results on ${ }^{266} \mathrm{Mt}$. Filled squares represent GSI data from the ${ }^{209} \mathrm{Bi}\left({ }^{58} \mathrm{Fe}, n\right)$ reaction [21], the open square represents LBNL results with the ${ }^{208} \mathrm{~Pb}\left({ }^{59} \mathrm{Co}, n\right)$ reaction. Horizontal error bars represent the energy width of the targets.

was obtained by fitting to $181 n$ cross section measurements, rather than the 12 that were available earlier, and has a second adjustable parameter reflecting drift in the asymmetry during the diffusion stage. For the ${ }^{58} \mathrm{Fe}+{ }^{209} \mathrm{Bi}$ reaction, the prediction of $12.8 \mathrm{pb}$ at a $\mathrm{CN}$ excitation energy of $\sim 13.5 \mathrm{MeV}$ is relatively close to the $1 n$ peak value of $7.5 \pm$ $2.7 \mathrm{pb}$ at approximately $13.4 \mathrm{MeV}$ from GSI's fit of the data. The correlation in excitation energies at the peak is excellent. The peak of their excitation function is approximately $3.5 \mathrm{MeV}$ below the predicted location of the barrier by the FBD model.

For the ${ }^{59} \mathrm{Co}+{ }^{208} \mathrm{~Pb}$ reaction, Świątecki predicts $7.1 \mathrm{pb}$ at $\sim 14.1 \mathrm{MeV} \mathrm{CN}$ excitation energy. Both values compare very well to the one data point obtained in this reaction, at 7.7 $7_{-3.3}^{+5.2} \mathrm{pb}$ and $14.9 \mathrm{MeV}$ excitation energy. The estimated location of the barrier is at $19.6 \mathrm{MeV}$, and our data point is approximately $4.7 \mathrm{MeV}$ below this barrier.

Because the exit channel for the two reactions is virtually identical (except for possible small differences in angular momenta and excitation energies), the nearly identical cross sections indicate nearly identical entrance channels. This is also the case in the reaction pairs producing ${ }^{258} \mathrm{Db}$ [15] and ${ }^{262} \mathrm{Bh}^{\mathrm{g}, \mathrm{m}}$ [13], with no statistically significant difference in the $1 n$ cross sections within each pair.

To determine if the cross section measured in the ${ }^{59} \mathrm{Co}+$ ${ }^{208} \mathrm{~Pb}$ reaction is truly at or very near the peak of the $1 n$ excitation function, a full excitation function would need to be measured. An additional 3-4 bombarding energies should be adequate to acquire the data needed for a more complete picture.

In conclusion, we have studied the new ${ }^{208} \mathrm{~Pb}\left({ }^{59} \mathrm{Co}, n\right){ }^{266} \mathrm{Mt}$ reaction with the 88-Inch Cyclotron and BGS at LBNL. A cross section of $7.7_{-3.3}^{+5.2} \mathrm{pb}$ was measured at a $\mathrm{CN}$ excitation energy of $14.9 \mathrm{MeV}$. The measured half-life of ${ }^{266} \mathrm{Mt}$ was $3.3_{-1.0}^{+2.5} \mathrm{~ms}$, corresponding well with the literature value of $1.7_{-0.4}^{+0.6} \mathrm{~ms}$. The decay properties of all daughter products agree well with previously published results.

We would like to thank D. Leitner and the operations staff of the 88-Inch Cyclotron for providing intense, stable beams of ${ }^{59} \mathrm{Co}$ for these experiments. The authors also wish to express their thanks to W. J. Świątecki for his theoretical predictions and stimulating discussion, and the target laboratory at GSI for the targets used in these studies. This work was supported in part by the Director, Office of High Energy and Nuclear Physics, Nuclear Physics Division of the U.S. Department of Energy, under contract DE-AC02-05CH11231.
[1] S. Hofmann, Rep. Prog. Phys. 61, 639 (1998).

[2] P. Armbruster, Acta Phys. Pol. B 34, 1825 (2003).

[3] G. Münzenberg et al., Z. Phys. A 300, 107 (1981).

[4] G. Münzenberg et al., Z. Phys. A 333, 163 (1989).

[5] G. Münzenberg et al., Z. Phys. A 317, 235 (1984).

[6] G. Münzenberg et al., Z. Phys. A 330, 435 (1988).

[7] S. Hofmann et al., Z. Phys. A 350, 281 (1995).

[8] K. Morita et al., J. Phys. Soc. Jpn. 73, 2593 (2004).

[9] W. J. Świątecki, K. Siwek-Wilcyńska, and J. Wilczyński, Acta Phys. Pol. B 34, 2049 (2003).

[10] W. J. Świątecki, K. Siwek-Wilcyńska, and J. Wilczyński, Phys. Rev. C 71, 014602 (2005).

[11] W. J. Świątecki (private communication, 2007).

[12] S. L. Nelson et al., Phys. Rev. Lett. 100, 022501 (2008).

[13] S. L. Nelson et al., Phys. Rev. C 78, 024606 (2008).

[14] I. Dragojević et al., Phys. Rev. C 78, 024605 (2008).

[15] J. M. Gates et al., Phys. Rev. C 78, 034604 (2008).

[16] C. M. Folden III et al., Phys. Rev. C 73, 014611 (2006).

[17] S. Hofmann et al., Eur. Phys. J. A 14, 147 (2002).

[18] K. Morita et al., J. Phys. Soc. Jpn. 73, 1738 (2004).

[19] C. M. Folden III et al., Phys. Rev. Lett. 93, 212702 (2004).

[20] G. Münzenberg et al., Z. Phys. A 315, 145 (1984).

[21] S. Hofmann et al., Z. Phys. A 358, 377 (1997).
[22] G. Münzenberg et al., Nucl. Instrum. Methods 161, 65 (1979).

[23] K. E. Gregorich and V. Ninov, Book of Abstracts, 219th ACS National Meeting, San Francisco, CA, 26-30 March 2000, NUCL.

[24] V. Ninov, K. E. Gregorich, and C. A. McGrath, AIP Conf. Proc. 455, 704 (1998).

[25] J. F. Ziegler, computer code SRIM-2003, available from http://www.srim.org, (2003).

[26] J. F. Ziegler, Nucl. Instrum. Methods B 219-220, 1027 (2004).

[27] W. D. Myers and W. J. Świątecki, Nucl. Phys. A601, 141 (1996).

[28] K. E. Gregorich et al., Phys. Rev. C 72, 014605 (2005).

[29] S. L. Nelson, Ph.D. thesis, University of California, Berkeley, Report No. LBNL-312E, 2008.

[30] K. H. Schmidt et al., Z. Phys. A 316, 19 (1984).

[31] G. Audi et al., Nucl. Phys. A729, 3 (2003).

[32] S. Hofmann et al., Eur. Phys. J. A 10, 5 (2001).

[33] F. P. Heßberger et al., Z. Phys. A 322, 557 (1985).

[34] F. P. Heßberger et al., Eur. Phys. J. A 12, 57 (2001).

[35] P. Eskola, Phys. Rev. C 7, 280 (1973).

[36] A. Ghiorso et al., Phys. Rev. C 7, 2032 (1973).

[37] S. Amiel et al., Phys. Rev. 106, 553 (1957).

[38] J. M. Gates et al., Phys. Rev. C 77, 034603 (2008).

[39] K. E. Gregorich et al., Phys. Rev. C 74, 044611 (2006). 